\title{
A Subjective Probability Based Deductive Approach to Global Trust Evaluation in Composite Services
}

\author{
Lei Li \\ Department of Computing \\ Macquarie University \\ Sydney, NSW 2109, Australia \\ lei.li@mq.edu.au
}

\author{
Yan Wang \\ Department of Computing \\ Macquarie University \\ Sydney, NSW 2109, Australia \\ yan.wang@mq.edu.au
}

\begin{abstract}
In Service-Oriented Computing (SOC) environments, the trustworthiness of each service provider is critical for a service client when selecting one from a large pool of service providers. The trust value of a service provider is usually in the range of $[0,1]$ and is evaluated from the ratings given by service clients, which represent the subjective belief of service clients on the satisfaction of delivered services. So a trust value can be taken as a subjective probability, by which one party believes that another party can perform an action in a certain situation. Hence, subjective probability theory should be adopted in trust evaluation. In addition, in SOC environments, a service provider usually can invoke the services from other service providers forming a composite service. Thus, the global trust of a composite service should be evaluated based on both the subjective probability property of trust and complex invocation structures.

In this paper, we first interpret the trust dependency caused by direct service invocations as conditional probability. Then, on the basis of trust dependency, we propose a SubjectivE probabiLity basEd deduCTIVE (SELECTIVE) approach to evaluate the subjective global trustworthiness of a composite service. All these processes follow subjective probability theory and keep the subjective probability property of trust in evaluations. Our experimental results demonstrate that when compared with existing approaches our proposed SELECTIVE approach can yield more reasonable results.
\end{abstract}

\section{INTRODUCTION}

In recent years, Service-Oriented Computing (SOC) has taken a central stage as an emerging research area attracting an increasing amount of attention. Thus, there is an increasing demand for information technologies to support the SOC architecture with a loose coupling between services to provide great flexibility for service applications [12].

Services are self-contained and modular applications that can be described, published, located and invoked in SOC environments [14]. However, to satisfy the specified functional requirement, it may have to integrate heterogeneous environments using an approach that links service providers together to build complex, yet manageable composite services [12]. In such an environment, in addition to functionality, trust is also a key factor for service client in service selection and composition [10].

Different from peer-to-peer (P2P) information-sharing networks or eBay system, where a binary rating system is adopted [7, 18], in SOC environments, a rating given by a service client is usually in or can be scaled to the range of $[0,1][7,9,15]$, which can represent the subjective belief of the service client on the satisfaction of a delivered service [10]. The trust value of a service provider can be obtained after evaluating trust ratings by trust management authorities. According to the definition of trust in $[7,10]$, if the trust value is in the range of $[0,1]$, it can be taken as the subjective probability by which one party expects that another party can perform a given action. Without any trust management mechanism, fraudulent service providers with attractive advertisements may be invoked by many service clients who subsequently become victims. These attacks may lead to unexpected service quality degradation and the monetary loss among service clients. Hence, the trust evaluation of services and service providers is highly desirable and critical to SOC applications.

However, in SOC environments, to satisfy the specified functionality requirement, a service provider may have to invoke the services from other service providers forming composite services leading to complex invocation structures and trust dependency. Thus, there is a necessity to evaluate the global trust of a composite service which has complex invocation structures over service components with different trust information. Although these compositions can certainly enrich service provision, they greatly increase the computational complexity. This makes a proper subjective global trust evaluation a real challenge and demands an effective algorithm for it.

In the literature, although there are a number of studies on the global trust evaluation of composite services $[8,10]$, some problems remain open.

1) According to the definition introduced in [7, 10], trust can be taken as a subjective probability, i.e., the degree of belief that an individual has in the truth of a proposition $[2,5]$, rather than a classical probability, which is the occurrence frequency of an event [3]. Hence, subjective probability theory [2, 5] should be adopted in trust evaluation.

2) In our previous work [9], it is pointed out that in composite services, the trust of a service component only depends on its own trust propensity and the trust of its direct predecessor(s). Namely, the trust dependency between service components results from direct invocations. In complex composite services consisting of varies atomic invocation structures, there are a large mount of indirect invocations. Thus, in the global trust evaluation of a composite 
service, it is still a big challenge to properly interpret the dependency caused by indirect invocations with subjective probability theory.

Although there are a variety of trust evaluation approaches existing in different areas [7, 18, 20], they either ignore the subjective probability property of trust ratings, or neglect complex invocation structures. As a result, no proper mechanism exists yet for evaluating the subjective global trustworthiness of a composite service.

In this paper, we first interpret the trust dependency caused by direct service invocations as conditional probability, which can be evaluated based on the subjective trust estimation with discrete subjective ratings of service components [9]. Then, on the basis of trust dependency, we propose a SubjectivE probabiLity basEd deduCTIVE (SELECTIVE) approach for subjective global trust evaluation in composite services. All these processes follow subjective probability theory and keep the subjective probability property of trust in evaluations.

This paper is organized as follows. Section II reviews existing studies in trust evaluation, service composition and service selection. Section III briefly introduces composite services with six atomic invocations, and the probability interpretation of trust dependency. Section IV presents our novel SubjectivE probabiLity basEd deduCTIVE (SELECTIVE) approach in composite services. Experiments are presented in Section $\mathrm{V}$ for further illustrating that when compared with existing approaches our proposed SELECTIVE approach can yield more reasonable results. Finally Section VI concludes our work.

\section{RELATED WORK}

The issue of trust has been widely studied in many applications. In e-commerce (EC) or SOC, the reputationbased trust value is a very important indication for a buyer or a service client to make a selection from a pool of sellers or service providers providing the same product or service, because the buyer or service client usually would like to order from the seller or service provider with the best transaction reputation [10]. This is particularly important when the buyer or service client has to select from unknown sellers or service providers [12]. In P2P information-sharing networks, it is quite natural for a client peer to doubt if a serving peer can provide the complete file prior to any download action, which may be quite overhead-consuming (e.g., time and network bandwidth).

As we have pointed out, trust is the subjective probability by which one party expects that another party performs a given action [7, 10], and it is better to adopt subjective probability theory in trust evaluation. In the early 20th century, the definition of subjective probability was first proposed [13]. The most initial work of subjective probability theory focused on Bayesian inference [13]. Later on, non-Bayesian inference methods in subjective probability theory were proposed and has attracted much more attention. For example, Jeffrey [5] proposes a causal decision theory to deal with the subjective decision- making, which is one of the most important non-Bayesian inference methods.

In the literature, there are some works to deal with subjective ratings [6, 16]. In [6], Jøsang proposes a framework for combining subjective ratings from different sources based on Dempster-Shafer belief theory, which is a generalization of the Bayesian theory of subjective probability. In [16], Wang et al. set up a bijection from subjective ratings to trust values with a mathematical understanding of subjective trust in multiagent systems. However, both models use either a binary rating (positive or negative) system or a triple rating (positive, negative or uncertain) system that is more suitable for securityoriented or P2P file-sharing trust management systems. In SOC environments, a rating as a numerical value in $[0,1]$ is more suitable [20].

In SOC applications, the criteria of service selection take into account not only functionality but also trust [11]. In the literature, a number of QoS-aware service selection mechanisms have been developed, aiming at QoS improvement in composite services [17, 21]. In [21], Zeng et al. present a QoS and reputation evaluation approach in composite services, and a service selection approach using linear programming techniques to find the optimal execution plan for composite services. In [17], Xiao et al. present an autonomic service provision framework for establishing QoS-assured end-to-end communication paths across domains. Their algorithms can provide QoS guarantees over domains.

There are also some works for trust-oriented service composition and selection [11, 19]. In [19], Xu et al. propose a reputation-enhanced QoS-based Web service discovery algorithm for service matching, ranking and selection based on existing Web service technologies. In [11], Malik et al. propose a set of decentralized techniques aiming at evaluating trust with ratings to facilitate trustoriented service selection and composition. Although these works adopt non-binary discrete ratings, neither the subjective probability property of trust nor service invocation structures has been taken into account.

Considering service invocation structures in composite services, in [8] Li and Wang propose a global trust evaluation approach. However, this approach has not taken the subjective probability property of trust into account. In [10], Li et al. propose a Bayesian inference based subjective trust evaluation approach which aggregates the subjective ratings from clients. Nevertheless, this approach still has some drawbacks. Firstly, it assumes that the trust ratings of each service component conform to a normal distribution, which is continuous. However, trust ratings adopted in most existing rating systems ${ }^{1,2,3}$ are discrete numbers. Thus, they cannot conform to a continuous distribution. Secondly, the proposed subjective probability approach (Bayesian inference) is to evaluate the trust values of service components, which is not used in the global trust evaluation of composite services. Therefore, although

\footnotetext{
${ }^{1}$ http://www.eBay.com/

${ }^{2}$ http://www.epinions.com/

${ }^{3}$ http://www.youtube.com/
} 


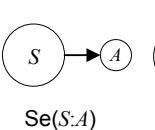

(a)

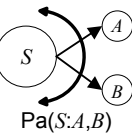

(b)

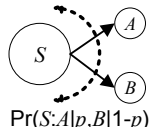

(c)

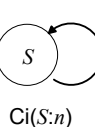

(d)

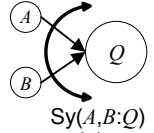

(e)

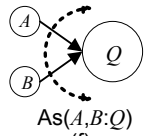

(f)

Figure 1. Atomic invocation relations

service invocation structures have been taken into account, the global trust evaluation of composite services does not keep the subjective probability property of trust. As in most existing rating systems ${ }^{1,2,3}$ trust ratings are discrete numbers, the numbers of the occurrences of all ratings of each service component conform to a multinomial distribution [9]. Hence, in [9] Li and Wang propose a subjective trust estimation approach for service components based on Bayesian inference, which can aggregate the non-binary discrete subjective ratings given by service clients and keep the subjective probability property of trust ratings and trust results. Although the joint subjective probability approach proposed in [9] considers the trust dependency between service components caused by direct invocations, it does not take into account the composition of trust dependency, which is caused by indirect invocations in composite services.

In the literature, there are some existing reliability evaluation approaches [2]. The similarity between trust evaluation and reliability evaluation is that they both take probabilities of components and the network structure of composite services or products into account. The difference of them is that trust evaluation is a subjective assessment and subjective probability theory should be adopted. In contrast, reliability evaluation is an objective assessment and classical probability theory should be adopted. In addition, in the literature, no reliability evaluation approach adopts the probability deductive process.

In summary, when considering the subjective probability property of trust and the composition of trust dependency, a proper subjective global trust evaluation approach is necessary and important for trust-oriented composite service selection. This is the focus of our work in this paper.

\section{Service InVocation And TRUst Dependency}

\section{A. Composite Service Structures}

A composite service is a conglomeration of services with invocations between them [10]. Six atomic invocation relations [10] in composite services are introduced below and are depicted in Fig. 1.

- Sequential Invocation: A service $S$ invokes its unique succeeding service $A$. It is denoted as $\operatorname{Se}(S: A)$ (see Fig. 1(a)).

- Parallel Invocation: A service $S$ invokes its succeeding services in parallel. E.g., if $S$ has successors $A$ and $B$, it is denoted as $\mathrm{Pa}(S: A, B)$ (see Fig. 1(b)).

- Probabilistic Invocation: A service $S$ invokes its succeeding services with certain probabilities. E.g., if $S$ invokes successors $A$ with probability $p$ and $B$ with probability $1-p$, it is denoted as $\operatorname{Pr}(S: A|p, B| 1-p)$ (see Fig. 1(c))

- Circular Invocation: A service $S$ invokes itself for $n$ times. It is denoted as $\mathrm{Ci}(S \mid n)$ (see Fig. 1(d)).

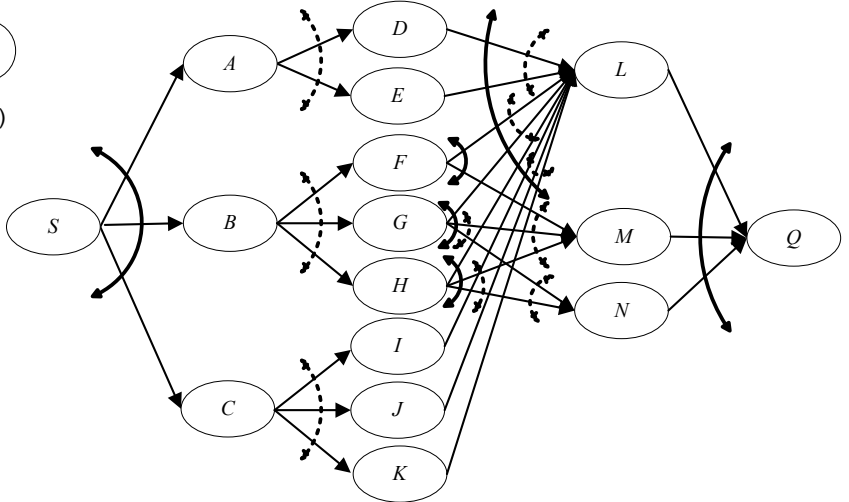

Figure 2. The service invocation graph $(S I G)$ for the travel plan

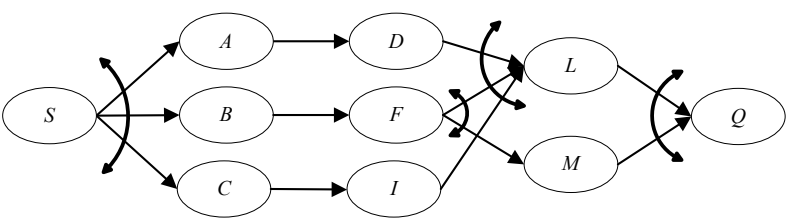

Figure 3. A service execution flow $(S E F)$ in the $S I G$

- Synchronous Activation: A service $Q$ is activated only when all its preceding services have been completed. E.g., if $Q$ has synchronous predecessors $A$ and $B$, it is denoted as $\operatorname{Sy}(A, B: Q)$ (see Fig. 1(e)).

- Asynchronous Activation: A service $Q$ is activated as the result of the completion of one of its preceding services. E.g., if $Q$ has asynchronous predecessors $A$ and $B$, it is denoted as $\operatorname{As}(A, B: Q)$ (see Fig. 1(f)).

Here we introduce an example of composite services. Example 1: Mr. Smith in Sydney, Australia is making a travel plan to attend an international conference in Washington DC, USA. His plan includes conference registration, airline from Sydney to Washington, accommodation and local transportation. Regarding conference registration $A$, Smith could pay online $D$ or by fax $E$ with a credit card $L$. Regarding accommodation reservation $B$, Smith could make a reservation at hotel $F, G$ or $H$ with credit card $L$. According to the hotel choice, Smith could arrange the local transportation, e.g., take a taxi $M$ to $F$, take a taxi $M$ or a bus $N$ to either $G$ or $H$. Regarding airplane booking $C$, Smith could choose from airlines $I, J$ and $K$ with the credit card $L$ for the payment.

In Example 1, starting from a service invocation root $S$ and ending at a service invocation terminal $Q$, the composite service consisting of all combinations of travel plans can be depicted by a service invocation graph $(S I G)$ in Fig. 2. Each feasible travel plan is termed as a service execution flow $(S E F)$, which is a subgraph of an SIG. An $S E F$ example of the $S I G$ in Fig. 2 is plotted in Fig. 3.

When a service client searches the optimal SEF with the maximal global trust value from multiple $S E F$ s in an $S I G$, a proper mechanism is necessary for the subjective global trust evaluation of an $S E F$ based on the trust ratings of service components and the invocations between service components. This global trust evaluation mechanism will be introduced in Section IV.

\section{B. Rating Determination of Service Component}

Based on subjective probability theory, the following definition is introduced to determine every rating of a 
Table I

RATINGS FOR SERVICE COMPONENTS IN THE SEF IN FIG. 3

\begin{tabular}{|c|c|c|c|c|c|c|c|c|c|c|c|}
\hline & $S$ & $A$ & $B$ & $C$ & $D$ & $F$ & $G$ & $I$ & $L$ & $M$ & $Q$ \\
\hline$e_{1}$ & 1 & 0.5 & 1 & 0.75 & 1 & 0.75 & 1 & 1 & 1 & 0.75 & 1 \\
\hline$e_{2}$ & 1 & 0.75 & 1 & 0.75 & 1 & 1 & 0.75 & 1 & 0.75 & 1 & 1 \\
\hline$e_{3}$ & 1 & 0.75 & 0.75 & 0.75 & 0.25 & 1 & 0 & 1 & 1 & 1 & 1 \\
\hline$e_{4}$ & 1 & 1 & 1 & 0.75 & 1 & 1 & 1 & 1 & 1 & 0.75 & 1 \\
\hline$e_{5}$ & 1 & 1 & 1 & 1 & 1 & 0.75 & 0.75 & 1 & 1 & 1 & 1 \\
\hline$e_{6}$ & 1 & 1 & 0.75 & 0.75 & 0.25 & 0.75 & 1 & 1 & 1 & 1 & 1 \\
\hline$e_{7}$ & 1 & 0.5 & 1 & 0.75 & 0 & 0.5 & 1 & 1 & 1 & 0.75 & 1 \\
\hline$e_{8}$ & 1 & 1 & 0.75 & 0.75 & 1 & 0.75 & 0.75 & 0.75 & 1 & 1 & 1 \\
\hline$e_{9}$ & 1 & 1 & 0.75 & 0.75 & 0.75 & 1 & 0.75 & 0.5 & 1 & 1 & 1 \\
\hline$e_{10}$ & 1 & 0.75 & 0.75 & 0.75 & 0.75 & 0.75 & 0.75 & 1 & 0.75 & 1 & 1 \\
\hline$e_{11}$ & 1 & 0.75 & 0.75 & 0.5 & 1 & 1 & 1 & 0.75 & 1 & 1 & 1 \\
\hline$e_{12}$ & 1 & 0.75 & 1 & 1 & 0.75 & 1 & 0 & 0.5 & 0.75 & 0.5 & 1 \\
\hline$e_{13}$ & 1 & 0.75 & 0.75 & 0.75 & 1 & 1 & 0.75 & 0 & 1 & 0.5 & 1 \\
\hline$e_{14}$ & 1 & 1 & 0.5 & 0.75 & 0.75 & 1 & 0.75 & 1 & 1 & 1 & 1 \\
\hline$e_{15}$ & 1 & 1 & 1 & 0.75 & 1 & 0.75 & 0.75 & 1 & 1 & 0.75 & 1 \\
\hline$e_{16}$ & 1 & 1 & 1 & 0.75 & 1 & 1 & 1 & 1 & 1 & 0.75 & 1 \\
\hline$e_{17}$ & 1 & 1 & 1 & 0.25 & 0.25 & 1 & 0 & 0.5 & 0.75 & 1 & 1 \\
\hline$e_{18}$ & 0.75 & 1 & 0.75 & 0.5 & 1 & 1 & 0.75 & 1 & 1 & 1 & 1 \\
\hline$e_{19}$ & 1 & 0.75 & 0.75 & 1 & 1 & 1 & 0.75 & 0.75 & 0.75 & 1 & 1 \\
\hline$e_{20}$ & 1 & 1 & 1 & 0.5 & 1 & 0.75 & 0.75 & 1 & 1 & 0.75 & 1 \\
\hline
\end{tabular}

Table II

TRUST ESTIMATION OF SERVICE COMPONENTS IN THE SEF IN FIG. 3 \begin{tabular}{|c|c|c|c|c|c|c|c|c|c|c|c|}
\hline & $S$ & $A$ & $B$ & $C$ & $D$ & $F$ & $G$ & $I$ & $L$ & $M$ & $Q$ \\
\hline$t$ & 0.80 & 0.65 & 0.64 & 0.42 & 0.43 & 0.68 & 0.52 & 0.45 & 0.76 & 0.67 & 0.82 \\
\hline$u$ & 0.20 & 0.35 & 0.36 & 0.56 & 0.49 & 0.32 & 0.35 & 0.52 & 0.24 & 0.33 & 0.18 \\
\hline$d$ & 0 & 0 & 0 & 0.02 & 0.08 & 0 & 0.13 & 0.03 & 0 & 0 & 0 \\
\hline
\end{tabular}

service component as trust, distrust or uncertain.

Definition 1: For each trust rating $r_{i} \in[0,1]$, we have

$$
r_{i} \text { is } \begin{cases}\text { trust, } & \text { if } r_{i} \geq 1-t, \\ \text { distrust, } & \text { if } r_{i} \leq d, \\ \text { uncertain, } & \text { if } d<r_{i}<1-t,\end{cases}
$$

where $t+d+u=1$, and trust $t$, distrust $d$ and uncertainty $u$ are estimated by Bayesian inference proposed in [9].

\section{Probability Interpretation of Trust Dependency}

Dependency is a state in which one object uses a functionality of another object [2]. In composite services, the dependency between service components results from direct invocations [9], e.g., if there is a direct invocation from service component $A$ to service component $B$, we say that " $B$ depends on $A$ ".

Definition 2: In composite services, trust dependency represents the fact that the trust of a service component is only dependent on its trust propensity and the trust of its direct predecessor(s).

According to the theorem about probabilities of conditionals and conditional probabilities [4], we can use conditional probability to formalize trust dependency.

Definition 3: In composite services, if $\left\{P_{i}\right\}$ are the direct predecessors of service component $S$, and $P$ is the rational subjective probability function, we have

$$
P\left(\bigwedge P_{i} \succeq S\right)=P\left(S \mid \bigwedge P_{i}\right) .
$$

In order to evaluate the conditional probability for trust dependency, in subjective probability theory [5], the following principle has been proposed for bridging from classic probability, i.e., the occurrence frequency of an event, to subjective probability, i.e., the degree of belief that an individual has in the truth of a proposition.

Principle 1: Without any additional knowledge, our knowledge that the chance of hypothesis $H$ has probability $p$ guarantees that our subjective probability for $H$ is $p$.
Table III

\begin{tabular}{|c|c|c|c|c|c|c|c|c|c|c|c|}
\hline & $S$ & $A$ & $B$ & $C$ & $D$ & $F$ & $G$ & $I$ & $L$ & $M$ & $Q$ \\
\hline$e_{1}$ & ust & ust & rust & trust & trust & rust & trust & trust & trust & trust & trust \\
\hline$e_{2}$ & ust & ust & trust & trust & trust & trust & trust & trust & trust & trust & trust \\
\hline$e_{3}$ & trust & trust & trust & trust & uncertain & trust & distrust & trust & trust & trust & trust \\
\hline$e_{4}$ & trust & trust & trust & trust & trust & trust & trust & trust & trust & trust & trust \\
\hline$e_{5}$ & trust & trust & trust & trust & trust & trust & trust & trust & trust & trust & trust \\
\hline$e_{6}$ & trust & trust & trust & trust & ncertain & trust & trust & trust & trust & trust & trust \\
\hline$e_{7}$ & trust & trust & trust & trust & distrust & trust & trust & trust & trust & trust & trust \\
\hline$e_{8}$ & trust & trust & trust & trust & trust & trust & trust & trust & trust & trust & trust \\
\hline$e_{9}$ & trust & trust & trust & trust & trust & trust & trust & uncertain & trust & trust & trust \\
\hline$e_{10}$ & trust & trust & trust & trust & trust & trust & trust & trust & trust & trust & trust \\
\hline$e_{11}$ & trust & trust & trust & uncertain & trust & trust & trust & trust & trust & trust & trust \\
\hline$e_{12}$ & trust & trust & trust & trust & trust & trust & distrust & uncertain & trust & trust & trust \\
\hline$e_{13}$ & trust & trust & trust & trust & trust & trust & trust & distrust & trust & trust & trust \\
\hline$e_{14}$ & trust & trust & trust & trust & trust & trust & trust & trust & trust & trust & trust \\
\hline$e_{15}$ & trust & trust & trust & trust & trust & trust & trust & trust & trust & trust & trust \\
\hline$e_{16}$ & trust & trust & trust & trust & trust & trust & trust & trust & trust & trust & trust \\
\hline$e_{17}$ & trust & trust & trust & uncertain & uncertain & trust & distrust & uncertain & trust & trust & trust \\
\hline$e_{18}$ & trust & trust & trust & uncertain & trust & trust & trust & trust & trust & trust & trust \\
\hline$e_{19}$ & trust & trust & trust & trust & trust & trust & trust & trust & trust & trust & trust \\
\hline$e_{20}$ & trust & trust & trust & uncertain & trust & trust & trust & trust & trust & trust & trust \\
\hline
\end{tabular}

TRUSTWORTHINESS OF EACH RATING IN TABLE I

Therefore, according to the definition of conditional probability, the trust dependency, which is the conditional probability of the trustworthiness of a service component given the trustworthiness of its predecessors, can be evaluated based on Principle 1. E.g., if $\left\{P_{i}\right\}$ are the direct predecessors of service component $S$, the evaluation of trust dependency of $\bigwedge P_{i} \succeq S$ is to evaluate $P\left(t_{S} \mid t_{\bigwedge P_{i}}\right)$, $P\left(t_{S} \mid u_{\wedge P_{i}}\right)$ and $P\left(t_{S} \mid d_{\wedge P_{i}}\right)$. In addition, as the service invocation root in an $S I G$ has no predecessor, its trust dependency can be evaluated directly from its ratings according to Principle 1.

Here we assume that when a rating of a delivered service is stored by the trust management authority, the invocation relationship (i.e., the predecessor(s) of the delivered service) is also recorded.

Example 2: Let's take the service execution flow ( $S E F)$ in Fig. 3 as an example. All ratings of service components in Fig. 3 are taken from Epinions ${ }^{2}$ and are listed in Table I, where each row corresponds to an execution of the $S E F$.

For service component $C$ in Fig. 3 with all its 20 trust ratings in column $C$ in Table I, according to Bayesian inference proposed in [9], we can obtain trust $t=0.42$, distrust $d=0.02$ and uncertainty $u=0.56$. Similarly, all these parameters for each service component in the $S E F$ in Fig. 3 can be obtained, as listed in Table II. Hereafter, for each rating $r_{C i}$ of $C$, according to Definition 1 , we have

$$
r_{C i} \text { is } \begin{cases}\text { trust, } & \text { if } r_{C i} \geq 0.58 \\ \text { distrust, } & \text { if } r_{C i} \leq 0.02 ; \\ \text { uncertain, } & \text { if } 0.02<r_{C i}<0.58\end{cases}
$$

Let $t_{Y}, d_{Y}$ and $u_{Y}$ denote the trust, distrust or uncertain of any service component $Y$ in the $S E F$ in Fig. 3, which are calculated and listed in Table III. As service component $C$ invokes service component $I$ (denoted as $C \succeq I$ ) in Fig. 3, following the definition of conditional probability, $P\left(t_{I} \mid t_{C}\right)$ is the chance of $I$ to be trust given its direct predecessor $C$ is trust. According to the results in Table III, there are 20 executions in total and 13 of them (i.e. $e_{1}-e_{8}, e_{10}, e_{14}-e_{16}$ and $\left.e_{19}\right)$ are the case that $I$ is trust given $C$ is trust. Hence, we have $P\left(t_{I} \mid t_{C}\right)=13 / 20=0.65$. 
Likewise, we can compute $P\left(t_{I} \mid u_{C}\right)=3 / 20=0.15$ and $P\left(t_{I} \mid d_{C}\right)=0 / 20=0$. Namely, the trust dependency of $C \succeq I$ has been evaluated. Following the same procedure, all the conditional probabilities corresponding to each trust dependency in an SEF can be evaluated and listed in Table IV.

\section{Subjective Probability Based Deductive (SELECTIVE) APPROACH For Global Trust Evaluation of Composite Services}

In this section, we propose a SubjectivE probabiLity basEd deduCTIVE (SELECTIVE) approach that evaluates the subjective global trustworthiness of an $S E F$ from all the trust dependency in the $S E F$. This process follows subjective probability theory and keeps the subjective probability property of trust in evaluations.

In subjective probability theory, there is causal decision theory [5]. In this section, we borrow its idea in dealing with subjective decision-making based on the imputations of probabilistic causal influence, but extend it to the evaluation of the subjective global trustworthiness of an $S E F$ in composite services.

On the basis of trust dependency caused by direct invocations, an indirect invocation also leads to a certain dependency relationship, which is actually the composition of trust dependency. For example, in Fig. 4, service component $A$ is dependent on service component $S$ and service component $B$ is dependent on $A$. Therefore, $B$ is dependent on $S$ indirectly, compositing trust dependency $S \succeq A$ and $A \succeq B$. As each trust dependency corresponds to a conditional probability, a composition of trust dependency can yield a subjective probability. In the process of composition, we can compute the probability of trustworthiness of a service component based on all its preceding trust dependency. Hence, the composition of trust dependency starting from the service invocation root to any intermediate service component in an $S E F$ until the service invocation terminal is reached can deductively compute the subjective probability of trustworthiness of the service invocation terminal based on all the trust dependency in the $S E F$, which is taken as the global trust value of the $S E F$.

Now let us illustrate the evaluation process of our SELECTIVE approach. The computational process starts from the service invocation root of an $S E F$ until the service invocation terminal is reached. For any intermediate service component $V$, when its direct predecessors $\left\{W_{i}\right\}$ are processed, we have $P\left(\bigwedge t_{W_{i}}\right)$ and $P\left(\neg \bigwedge t_{W_{i}}\right)$. In addition, trust dependency $P\left(t_{V} \mid \bigwedge t_{W_{i}}\right)$ and $P\left(t_{V} \mid \neg \bigwedge t_{W_{i}}\right)$ can be evaluated following the approach illustrated in Example 2. Then according to the law of total expectation [5] in causal decision theory, we compute

$P\left(t_{V}\right)=P\left(t_{V} \mid \bigwedge t_{W_{i}}\right) P\left(\bigwedge t_{W_{i}}\right)+P\left(t_{V} \mid \neg \bigwedge t_{W_{i}}\right) P\left(\neg \bigwedge t_{W_{i}}\right)$.

When the service invocation terminal is finally processed, the deducted subjective trust value results from the composition of all trust dependency from the service invocation root to the service invocation terminal in the $S E F$. Hence, it is taken as the subjective global trust value

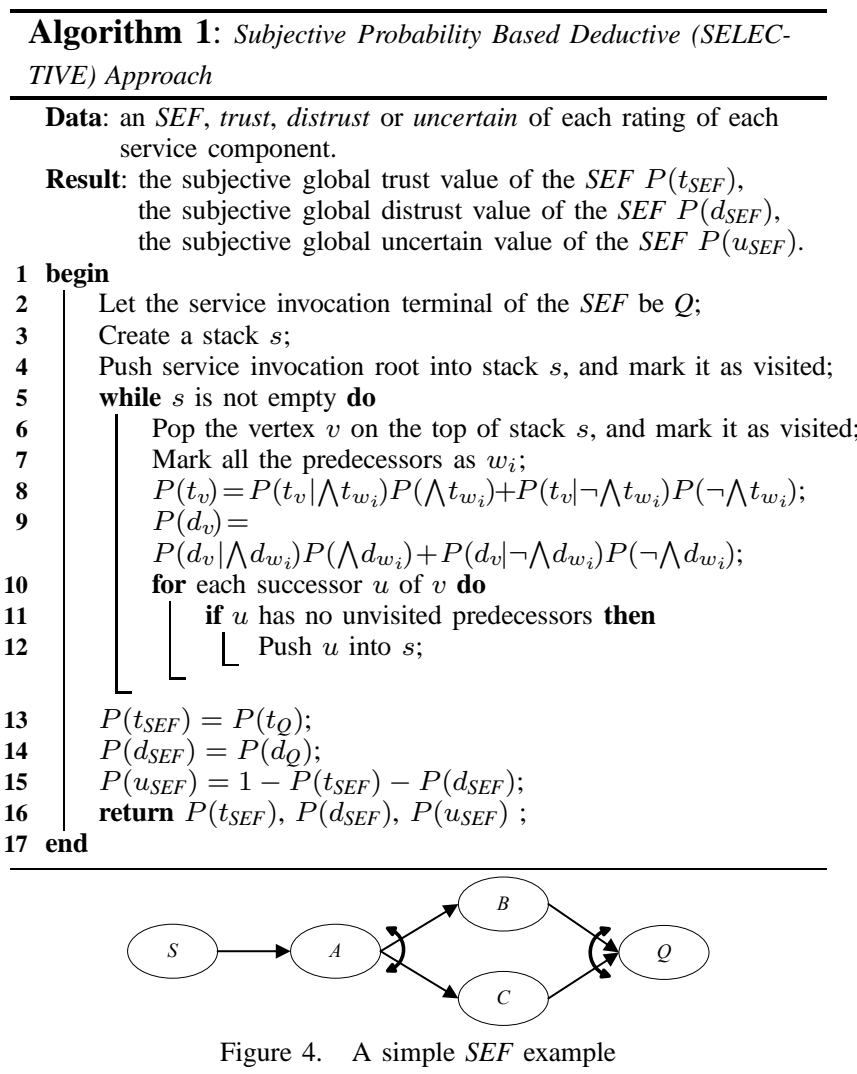

of the SEF. Similarly, the subjective global distrust value and the subjective global uncertain value can be obtained. The details of our SELECTIVE approach are presented in Algorithm 1, which extends the topological sort algorithm [1] that guarantees any node in a directed graph is always visited after all its predecessors. This algorithm incurs a complexity of $O\left(N_{v}+N_{e}\right)$, where $N_{v}$ is the number of service components (vertices) in an $S E F$ and $N_{e}$ is the number of invocations (edges).

Example 3: Let's illustrate the computational details of our proposed SELECTIVE approach with the simple $S E F$ in Fig. 4. As we have defined in Example 2, let $t_{A}$, $d_{A}$ and $u_{A}$ denote trust, distrust or uncertain of service component $A$ respectively. In Fig. 4, we can observe that service invocation root $S$ doesn't invoke service invocation terminal $Q$ directly, then there is no trust dependency of $Q$ on $S$ directly. However, with service components $A, B$ and $C, Q$ can be indirectly invoked by $S$, corresponding to the composition of trust dependency from $S$ to $Q$.

In Fig. 4 as $A$ is only dependent on $S$, and $t_{S}, d_{S}$ and $u_{S}$ are the partition of the trust space for $S$, according to the law of total expectation, we have

$$
\begin{aligned}
P\left(t_{A}\right) & =P\left(t_{A} \mid t_{S}\right) P\left(t_{S}\right)+P\left(t_{A} \mid d_{S}\right) P\left(d_{S}\right)+P\left(t_{A} \mid u_{S}\right) P\left(u_{S}\right) \\
& =P\left(t_{A} \mid t_{S}\right) P\left(t_{S}\right)+P\left(t_{A} \mid d_{S} \vee u_{S}\right)\left(1-P\left(t_{S}\right)\right) \\
& =P\left(t_{A} \mid t_{S}\right) P\left(t_{S}\right)+P\left(t_{A} \mid \neg t_{S}\right)\left(1-P\left(t_{S}\right)\right)
\end{aligned}
$$

where $\neg$ is the NOT operator in logic $P(\neg X)=1-P(X)$, $P\left(\neg t_{S}\right)=1-P\left(t_{S}\right)=P\left(d_{S}\right)+P\left(u_{S}\right)$. In addition, as $S$ is the service invocation root, $P\left(t_{S}\right), P\left(d_{S}\right)$ and $P\left(u_{S}\right)$ can be computed from the trust ratings of $S$ (as illustrated in Example 2). Moreover, trust dependency $P\left(t_{A} \mid t_{S}\right)$ and $P\left(t_{A} \mid \neg t_{S}\right)$ can also be computed (as illustrated in Example 
2). Hence, according to Eq. (4), $P\left(t_{A}\right)$ can be obtained. Similarly, $P\left(t_{B}\right)$ and $P\left(t_{C}\right)$ can be deducted as follows,

$$
\begin{aligned}
& P\left(t_{B}\right)=P\left(t_{B} \mid t_{A}\right) P\left(t_{A}\right)+P\left(t_{B} \mid \neg t_{A}\right)\left(1-P\left(t_{B}\right)\right),(5) \\
& P\left(t_{C}\right)=P\left(t_{C} \mid t_{A}\right) P\left(t_{A}\right)+P\left(t_{C} \mid \neg t_{A}\right)\left(1-P\left(t_{C}\right)\right)(6)
\end{aligned}
$$

As $P\left(t_{B} \mid t_{A}\right), P\left(t_{B} \mid \neg t_{A}\right), P\left(t_{C} \mid t_{A}\right)$ and $P\left(t_{C} \mid \neg t_{A}\right)$ in Eqs. (5) and (6) can be computed (as illustrated in Example 2), $P\left(t_{B}\right)$ and $P\left(t_{C}\right)$ can be obtained respectively. In Fig. 4 , as the service invocation terminal $Q$ is dependent on service components $B$ and $C$, we have

$$
\begin{aligned}
P\left(t_{Q}\right)= & P\left(t_{Q} \mid t_{B} \wedge t_{C}\right) P\left(t_{B} \wedge t_{C}\right) \\
& +P\left(t_{Q} \mid \neg\left(t_{B} \wedge t_{C}\right)\right) P\left(\neg\left(t_{B} \wedge t_{C}\right)\right) .
\end{aligned}
$$

In addition, as $B$ and $C$ are independent of each other, $P\left(t_{B} \wedge t_{C}\right)=P\left(t_{B}\right) P\left(t_{C}\right)$. Hence, from Eq. (7) we have

$$
\begin{aligned}
P\left(t_{Q}\right)= & P\left(t_{Q} \mid t_{B} \wedge t_{C}\right) P\left(t_{B}\right) P\left(t_{C}\right) \\
& +P\left(t_{Q} \mid \neg\left(t_{B} \wedge t_{C}\right)\right)\left(1-P\left(t_{B}\right) P\left(t_{C}\right)\right) .
\end{aligned}
$$

In Eq. (8), as both $P\left(t_{Q} \mid t_{B} \wedge t_{C}\right)$ and $P\left(t_{Q} \mid \neg\left(t_{B} \wedge t_{C}\right)\right)$ can be computed (as illustrated in Example 2), $P\left(t_{Q}\right)$ can be obtained. Therefore, the global trust of the $S E F, P\left(t_{S E F}\right)=$ $P\left(t_{Q}\right)$, can be evaluated from the above deductive process from Eq. (4) to Eq. (8).

When applying Algorithm 1 to the SEF in Fig. 3, we have the following process in steps.

Step 1: $P\left(t_{A}\right)=P\left(t_{A} \mid t_{S}\right) P\left(t_{S}\right)+P\left(t_{A} \mid \neg t_{S}\right)\left(1-P\left(t_{S}\right)\right)$;

Step 2: $P\left(t_{B}\right)=P\left(t_{B} \mid t_{S}\right) P\left(t_{S}\right)+P\left(t_{B} \mid \neg t_{S}\right)\left(1-P\left(t_{S}\right)\right)$;

Step 3: $P\left(t_{C}\right)=P\left(t_{C} \mid t_{S}\right) P\left(t_{S}\right)+P\left(t_{C} \mid \neg t_{S}\right)\left(1-P\left(t_{S}\right)\right)$;

Step 4: $P\left(t_{D}\right)=P\left(t_{D} \mid t_{A}\right) P\left(t_{A}\right)+P\left(t_{D} \mid \neg t_{A}\right)\left(1-P\left(t_{A}\right)\right)$;

Step 5: $P\left(t_{F}\right)=P\left(t_{F} \mid t_{B}\right) P\left(t_{B}\right)+P\left(t_{F} \mid \neg t_{B}\right)\left(1-P\left(t_{B}\right)\right)$;

Step 6: $P\left(t_{I}\right)=P\left(t_{I} \mid t_{C}\right) P\left(t_{C}\right)+P\left(t_{I} \mid \neg t_{C}\right)\left(1-P\left(t_{C}\right)\right)$;

Step 7: $P\left(t_{L}\right)=P\left(t_{L} \mid t_{D} \wedge t_{F} \wedge t_{I}\right) P\left(t_{D}\right) P\left(t_{F}\right) P\left(t_{I}\right)$

$$
+P\left(t_{L} \mid \neg\left(t_{D} \wedge t_{F} \wedge t_{I}\right)\right)\left(1-P\left(t_{D}\right) P\left(t_{F}\right) P\left(t_{I}\right)\right) ;
$$

Step 8: $P\left(t_{M}\right)=P\left(t_{M} \mid t_{F}\right) P\left(t_{F}\right)+P\left(t_{M} \mid \neg t_{F}\right)\left(1-P\left(t_{F}\right)\right) ;(16)$

Step 9: $P\left(t_{Q}\right)=P\left(t_{Q} \mid t_{L} \wedge t_{M}\right) P\left(t_{L}\right) P\left(t_{M}\right)$

$$
+P\left(t_{Q} \mid \neg\left(t_{L} \wedge t_{M}\right)\right)\left(1-P\left(t_{L}\right) P\left(t_{M}\right)\right) ;
$$

Step 10: $P\left(t_{\text {SEF }}\right)=P\left(t_{Q}\right)$.

As each trust dependency represented by a conditional probability in the above equations can be computed (as illustrated in Example 2), from Eq. (9) to Eq. (18), the subjective global trust value of the SEF in Fig. 3, $P\left(t_{S E F}\right)$, can be finally obtained. Similarly, the subjective global distrust value $P\left(d_{S E F}\right)$ of the $S E F$ in Fig. 3 can be computed, with which the subjective global uncertain value $P\left(u_{S E F}\right)=1-P\left(t_{S E F}\right)-P\left(d_{S E F}\right)$ can be obtained.

Regarding the comparison of multiple $S E F \mathrm{~s}$ with the same functionality, we firstly compare their trust values. For any two $S E F$ s, the one with a larger trust value is more preferable. If they have approximately the same trust value, their distrust values should be compared. The $S E F$ with a lower distrust value is more preferable.

Given $S E F_{1}$ with $\left(P\left(t_{S E F_{1}}\right), P\left(d_{S E F_{1}}\right), P\left(u_{S E F_{1}}\right)\right)$ and $S E F_{2}$ with $\left(P\left(t_{S E F_{2}}\right), P\left(d_{S E F_{2}}\right), P\left(u_{S E F_{2}}\right)\right)$, they are comparable in the following cases:
Table IV

TRUST DEPENDENCY IN THE SEF IN FIG. 3

\begin{tabular}{|c|c|c|c|c|c|}
\hline$P\left(t_{A} \mid t_{S}\right)$ & 1 & $P\left(t_{B} \mid t_{S}\right)$ & 1 & $P\left(t_{L} \mid t_{D} \wedge t_{F} \wedge t_{I}\right)$ & 0.65 \\
\hline$P\left(t_{A} \mid \neg t_{S}\right)$ & 0 & $P\left(t_{B} \mid \neg t_{S}\right)$ & 0 & $P\left(t_{L} \mid \neg\left(t_{D} \wedge t_{F} \wedge t_{I}\right)\right)$ & 0.35 \\
\hline$P\left(t_{C} \mid t_{S}\right)$ & 0.8 & $P\left(t_{D} \mid t_{A}\right)$ & 0.8 & $P\left(t_{Q} \mid t_{L} \wedge t_{M}\right)$ & 1 \\
\hline$P\left(t_{C} \mid \neg t_{S}\right)$ & 0 & $P\left(t_{D} \mid \neg t_{A}\right)$ & 0 & $P\left(t_{Q} \mid \neg\left(t_{L} \wedge t_{M}\right)\right)$ & 0 \\
\hline$P\left(t_{F} \mid t_{B}\right)$ & 1 & $P\left(t_{I} \mid t_{C}\right)$ & 0.65 & $P\left(t_{M} \mid t_{F}\right)$ & 1 \\
\hline$P\left(t_{F} \mid \neg t_{B}\right)$ & 0 & $P\left(t_{I} \mid \neg t_{C}\right)$ & 0.15 & $P\left(t_{M} \mid \neg t_{F}\right)$ & 0 \\
\hline \hline$P\left(d_{A} \mid d_{S}\right)$ & 0 & $P\left(d_{B} \mid d_{S}\right)$ & 0 & $P\left(d_{L} \mid d_{D} \wedge d_{F} \wedge d_{I}\right)$ & 0 \\
\hline$P\left(d_{A} \mid \neg d_{S}\right)$ & 0 & $P\left(d_{B} \mid \neg d_{S}\right)$ & 0 & $P\left(d_{L} \mid \neg\left(d_{D} \wedge d_{F} \wedge d_{I}\right)\right)$ & 0 \\
\hline$P\left(d_{C} \mid d_{S}\right)$ & 0 & $P\left(d_{D} \mid d_{A}\right)$ & 0 & $P\left(d_{Q} \mid d_{L} \wedge d_{M}\right)$ & 0 \\
\hline$P\left(d_{C} \mid \neg d_{S}\right)$ & 0 & $P\left(d_{D} \mid \neg d_{A}\right)$ & 0.05 & $P\left(d_{Q} \mid \neg\left(d_{L} \wedge d_{M}\right)\right)$ & 0 \\
\hline$P\left(d_{F} \mid d_{B}\right)$ & 0 & $P\left(d_{I} \mid d_{C}\right)$ & 0 & $P\left(d_{M} \mid d_{F}\right)$ & 0 \\
\hline$P\left(d_{F} \mid \neg d_{B}\right)$ & 0 & $P\left(d_{I} \mid \neg d_{C}\right)$ & 0.05 & $P\left(d_{M} \mid \neg d_{F}\right)$ & 0 \\
\hline
\end{tabular}

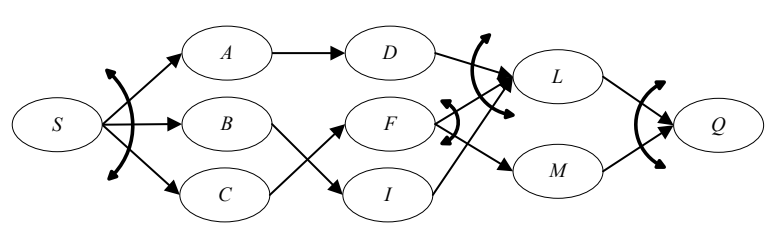

Figure 5. Service execution flow $S_{E F}$

Case 1: If $\left|P\left(t_{S E F_{1}}\right)-P\left(t_{S E F_{2}}\right)\right|<\epsilon_{1}$ and $\mid P\left(d_{S E F_{1}}\right)-$ $P\left(d_{S E F_{2}}\right) \mid<\epsilon_{2}, S E F_{1}$ and $S E F_{2}$ are equivalent in trust level, where $0<\epsilon_{1}, \epsilon_{2} \ll 1$ are thresholds that can be specified by service clients or trust management authorities.

Case 2: If $P\left(t_{S E F_{1}}\right)-P\left(t_{S E F_{2}}\right)>\epsilon_{1}, S E F_{1}$ is more preferable.

Case 3: If $\left|P\left(t_{S E F_{1}}\right)-P\left(t_{S E F_{2}}\right)\right|<\epsilon_{1}$ and $P\left(d_{S E F_{1}}\right)-$ $P\left(d_{S E F_{2}}\right)>\epsilon_{2}, S E F_{2}$ is more preferable.

\section{EXPERIMENTS}

In this section, we present the experimental results for studying our proposed SELECTIVE approach and explaining the following questions:

Q1: why service invocation structures should be taken into account in trust evaluation?

Q2: why trust dependency caused by direct invocations should be taken into account in global trust evaluation?

Q3: why the dependency caused by indirect invocations should be taken into account in trust evaluation?

In these experiments, ratings are taken from Epinions ${ }^{2}$, which is a popular online reputation system, and each

(18) rating is an integer in $\{1,2,3,4,5\}$. After normalization, a rating is in $\{0,0.25,0.5,0.75,1\}$. We set $\epsilon_{1}=\epsilon_{2}=0.001$ which are the thresholds in the comparison of multiple $S E F \mathrm{~s}$.

\section{A. Experiment 1 on Service Invocation Structure}

In this experiment, we take the $S E F$ in Fig. 3 (denoted as $S E F_{1}$ ) as an example to illustrate the computational details of our proposed SELECTIVE approach. By comparing our SELECTIVE approach with the existing global trust evaluation approach in [21], we explain why service invocation structures should be taken into account in trust evaluation (Q1).

As illustrated in Example 2 and listed in Table III, each rating of a service component can be judged as trust, distrust or uncertain. Then the trust dependency in composite services can be evaluated, as illustrated in Example 2 and listed in Table IV. According to Definition 2 , the trust of service invocation root $S$ can be computed 
Table V

TRUST RESULTS IN EXPERIMENT 1

\begin{tabular}{|c|c|c|}
\hline & $S E F_{1}$ & $S E F_{2}$ \\
\hline $\begin{array}{c}\text { Global trust evaluation } \\
\text { approach in [21] }\end{array}$ & $T_{S E F_{1}}=0.8763$ & $T_{S E F_{2}}=0.8763$ \\
\hline SELECTIVE approach & $P\left(t_{S E F_{1}}\right)=0.4820$ & $P\left(t_{S E F_{2}}\right)=0.3268$ \\
\hline
\end{tabular}

based on its ratings directly, and $P\left(t_{S}\right)=1$. Following our proposed SELECTIVE approach, the subjective global trust value of $S E F_{1}$ in Fig. 3, $P\left(t_{S E F_{1}}\right)$, can be calculated according to Eqs. (9) to (18). Hence, we can obtain $P\left(t_{S E F_{1}}\right)=0.4820$. Similarly, we have $P\left(d_{S E F_{1}}\right)=0$ and $P\left(u_{S E F_{1}}\right)=1-P\left(t_{S E F_{1}}\right)-P\left(d_{S E F_{1}}\right)=0.5180$.

In order to illustrate the necessity of service invocation structures in trust evaluation, let us change the service invocation structure of $S E F_{1}$ in Fig. 3 to be the one in Fig. 5, which is denoted as $S E F_{2}$. The difference between $S E F_{1}$ and $S E F_{2}$ is that in Fig. 3 service component $B$ invokes $F$ and $C$ invokes $I$. In contrast, in Fig. 5, $B$ invokes $I$ and $C$ invokes $F$. The trust dependency in $S E F_{2}$ can be evaluated as $P\left(t_{B} \mid t_{S}\right)=0.8, P\left(t_{B} \mid \neg t_{S}\right)=0$, $P\left(t_{C} \mid t_{S}\right)=1, P\left(t_{C} \mid \neg t_{S}\right)=0, P\left(t_{F} \mid t_{B}\right)=0.8, P\left(t_{F} \mid \neg t_{B}\right)=$ $0.2, P\left(t_{I} \mid t_{C}\right)=0.8, P\left(t_{I} \mid \neg t_{C}\right)=0$, and the rest trust dependency in $S E F_{2}$ is listed in Table IV. Following our SELECTIVE approach (from Eq. (9) to Eq. (18)), the subjective global trust value of $S E F_{2}$ is $P\left(t_{S E F_{2}}\right)=0.3268$. When compared with $P\left(t_{S E F_{1}}\right)=0.4820$, we can observe that following our SELECTIVE approach, we prefer $S E F_{1}$ to $S E F_{2}$ (also refer to Table V).

In most existing global trust evaluation approaches, service invocation structures have not been taken into account (e.g., the approach proposed in [21]). When we change the service invocation structure (e.g., change the invocation structure in Fig. 3 to be the one in Fig. 5), the global trust value is kept unchanged in these approaches (e.g., $T_{S E F_{1}}=T_{S E F_{2}}=0.8763$ (refer to Table V)). However, considering trust dependency, when we have changed the service invocation structure, the subjective global trust value of the $S E F$ should change as well (e.g., $P\left(t_{S E F_{1}}\right) \neq P\left(t_{S E F_{2}}\right)$ ). Hence, without considering service invocation structures, the trustworthiness of these two different $S E F$ s cannot be distinguished. Therefore, service invocation structures should be taken into account in the global trust evaluation of composite services, and our proposed SELECTIVE approach considering service invocation structures can yield reasonable results.

\section{B. Experiment 2 on Trust Dependency}

In this section, we present the experimental results to compare our proposed SELECTIVE approach with the existing global trust evaluation approach in [10], and explain why trust dependency should be taken into account in global trust evaluation (Q2).

In order to illustrate the necessity of trust dependency in trust evaluation, let us exchange the rating at execution $e_{9}$ (i.e., $\left.r_{I 9}=0.5\right)$ and the rating at $e_{18}$ (i.e., $r_{I 18}=1$ ) for service component $I$ in $S E F_{1}$, and let $S E F_{3}$ denote the exchanged $S E F$. Although the global trust evaluation approach proposed in [10] have considered service invocation structures, the trust dependency in composite services is ignored. In this experiment, without loss of generality, the weights of service components in all $\mathrm{Pa}$
Table VI

TRUST RESULTS IN EXPERIMENT 2

\begin{tabular}{|c|c|c|}
\hline & $S E F_{1}$ & $S E F_{3}$ \\
\hline $\begin{array}{c}\text { Global trust evaluation } \\
\text { approach in [10] }\end{array}$ & $T_{S E F_{1}}=0.1759$ & $T_{S E F_{3}}=0.1759$ \\
\hline SELECTIVE approach & $P\left(t_{S E F_{1}}\right)=0.4820$ & $P\left(t_{S E F_{3}}\right)=0.4892$ \\
\hline
\end{tabular}

Table VII

TRUST RESULTS IN EXPERIMENT 3

\begin{tabular}{|c|c|c|}
\hline & $S E F_{1}$ & $S E F_{2}$ \\
\hline $\begin{array}{c}\text { Joint subjective probability } \\
\text { approach in [9] }\end{array}$ & $P\left(S E F_{1}\right)=0.2704$ & $P\left(S E F_{4}\right)=0.2774$ \\
\hline SELECTIVE approach & $P\left(t_{S E F_{1}}\right)=0.4820$ & $P\left(t_{S E F_{4}}\right)=0.3537$ \\
\hline
\end{tabular}

structures of composite services are set to be 1 , and the requesting client's prior subjective belief about the trust of each service component is set to be $\delta=0.5$. Then, following Algorithm 1 in [10], the global trust value can be computed as $T_{S E F_{1}}=T_{S E F_{3}}=0.1759$ (also refer to Table VI).

However, when compared with the trust dependency in $S E F_{1}$, the trust dependency in $S E F_{3}$ changes to $P\left(t_{I} \mid t_{C}\right)=$ 0.7 and $P\left(t_{I} \mid \neg t_{C}\right)=0.1$. Following our proposed SELECTIVE approach, the subjective global trust value of $S E F_{3}$ is $P\left(t_{S E F_{3}}\right)=0.4892$, which is larger than $P\left(t_{S E F_{1}}\right)=$ 0.4820 (also refer to Table VI). This means following our SELECTIVE approach we prefer $S E F_{3}$ to $S E F_{1}$.

In the global trust evaluation approach proposed in [10], firstly the trust value of each service component is computed. Then based on service invocation structures, these values are aggregated to obtain the global trust value. Obviously, this approach has not considered trust dependency. However, following Definition 2, the trust of a service component is different when it is invoked by different direct predecessors. In order to consider this kind of dependency caused by invocations, when evaluating the global trust of composite services, it is necessary to take into account the trust dependency between service components, instead of a single trust value of every service component only. Therefore, our proposed SELECTIVE approach, which takes trust dependency into account, can yield reasonable results in the global trust evaluation of composite services.

\section{Experiment 3 on Trust-Oriented Composite Service Selection}

In this experiment, we compare our proposed SELECTIVE approach with the joint subjective probability approach proposed in [9] by applying both approaches to the travel plan composite services in Fig. 2. The joint subjective probability approach is proposed to evaluate the subjective global trust of a composite service. In this approach, we explain why the dependency caused by indirect invocations should be taken into account in trust evaluation $(\mathrm{Q} 3)$.

Let $S E F_{1}$ denote the service execution flow $\{S, A, B$, $C, D, F, I, L, M$ and $Q\}$ and let $S E F_{4}$ denote the service execution flow $\{S, A, B, C, D, G, I, L, M$ and $Q\}$ in Fig. 2. The difference of the two $S E F$ s is that $F$ is in $S E F_{1}$ and $\mathrm{G}$ is in $S E F_{4}$. All the ratings of service components in this section are taken from Epinions ${ }^{2}$ and are listed in Table I. For the sake of simplicity, we assume that the ratings of 
$L$ and $M$ when being invoked by $G$ are the same as their ratings when being invoked by $F$.

Following the joint subjective probability approach, with the trust dependency listed in Table IV, the subjective global trust value of $S E F_{1}$ can be obtained as $P\left(S E F_{1}\right)=$ 0.2704. In $S E F_{4}$, the trust dependency related to service component $G$ can be evaluated (as illustrated in Example 2). Hence, we have $P\left(t_{G} \mid t_{B}\right)=0.85, P\left(t_{G} \mid \neg t_{B}\right)=0$, $P\left(t_{L} \mid t_{D} \wedge t_{G} \wedge t_{I}\right)=0.6, P\left(t_{L} \mid \neg\left(t_{D} \wedge t_{G} \wedge t_{I}\right)\right)=0.4$, $P\left(t_{M} \mid t_{G}\right)=0.85$ and $P\left(t_{M} \mid \neg t_{G}\right)=0.15$, and the rest trust dependency in $S E F_{4}$ can be evaluated, as listed in Table IV. Following the joint subjective probability approach [9], we have $P\left(\mathrm{SEF}_{4}\right)=0.2774$, which is larger than $P\left(S E F_{1}\right)=0.2704$ (also refer to Table VII).

In contrast, following our proposed SELECTIVE approach, as we have illustrated in the first experiment, the subjective global trust value of $S E F_{1}$ is $P\left(t_{S E F_{1}}\right)=0.4820$. Similarly, the subjective global trust value of $S E F_{4}$ is $P\left(t_{S E F_{4}}\right)=0.3537$, which is smaller than $P\left(t_{S E F_{1}}\right)=$ 0.4820 (also refer to Table VII).

Both approaches consider both service invocation structures and trust dependency. However, in the above two examples, they yield completely different conclusions. That is because the joint subjective probability approach only considers trust dependency $P\left(t_{V} \mid \wedge t_{W_{i}}\right)$, i.e. the possibility of all service invocations in a composite service are trustworthy, where $\left\{W_{i}\right\}$ are the direct predecessors of $V$. In contrast, according to the law of total expectation in causal decision theory, our SELECTIVE approach takes into account not only trust dependency $P\left(t_{V} \mid \wedge t_{W_{i}}\right)$, but also trust dependency $P\left(t_{V} \mid \neg \bigwedge t_{W_{i}}\right)$, which contains both $P\left(t_{V} \mid \bigvee u_{W_{i}}\right)$ and $P\left(t_{V} \mid \bigvee d_{W_{i}}\right)$. In our SELECTIVE approach, every service component in a composite service corresponds to a subjective probability value, which is computed based on all its preceding trust dependency. Thus, the service invocation terminal corresponds to a subjective probability value. This value is computed based on all trust dependency in the composite service, caused by both direct invocations and indirect ones, and it is taken as the global trust value of the composite service. Therefore, our SELECTIVE approach can compute more reasonable results that can reflect the global trustworthiness of a composite service.

\section{CONCLUSIONS}

In this paper, firstly, the trust dependency caused by a direct invocation is interpreted as a conditional probability, which is evaluated based on the subjective trust estimation with discrete subjective ratings of service components. Then, we specify a subjective probability of each service component based on all its preceding trust dependency to evaluate the composition of trust dependency, which is caused by an indirect invocation. This probability interpretation provides a feasible means to deal with invocation structures with subjective probability theory. Furthermore, on the basis of trust dependency, a subjective probability based deductive approach has been proposed to evaluate the subjective global trustworthiness of a composite service. Experiments have demonstrated that our approach can deliver reasonable results that are critical for the decision-making of service clients in trustworthy service selection.

In composite services, if there is a dependency between QoS (Quality of Service) of service components, our SELECTIVE approach can be applied in QoS-oriented composite service selection. In our future work, with our subjective global trust evaluation approach, efficient algorithms will be studied for trust-oriented composite service selection.

\section{REFERENCES}

[1] J. Gross and J. Yellen. Handbook of Graph Theory. CRC Press, 2003.

[2] M. S. Hamada, A. G. Wilson, C. S. Reese, and H. F. Martz. Bayesian Reliability. Springer, 2008.

[3] W. W. Hines, D. C. Montgomery, D. M. Goldsman, and C. M. Borror. Probability and Statistics in Engineering. John Wiley \& Sons, Inc, 2003.

[4] A. Hójek. Probability, Logic, and Probability Logic, pages 362-384. The Blackwell Guide to Philosophical Logic. Blackwell Publishing, 2001.

[5] R. Jeffrey. Subjective Probability: The Real Thing. Cambridge University Press, April 2004.

[6] A. Jøsang. Subjective evidential reasoning. In IPMU, 2002.

[7] A. Jøsang, R. Ismail, and C. Boyd. A survey of trust and reputation systems for online service provision. Decision Support Systems, 43(2):618-644, 2007.

[8] L. Li and Y. Wang. Trust evaluation in composite services selection and discovery. In IEEE SCC 2009, pages 482$485,2009$.

[9] L. Li and Y. Wang. Subjective trust inference in composite services. In AAAI 2010, pages 1377-1384, 2010.

[10] L. Li, Y. Wang, and E.-P. Lim. Trust-oriented composite service selection and discovery. In ICSOC/ServiceWave 2009, pages 50-67, 2009.

[11] Z. Malik and A. Bouguettaya. RATEWeb: Reputation assessment for trust establishment among web services. VLDB J., 18(4):885-911, 2009.

[12] M. P. Papazoglou, P. Traverso, S. Dustdar, and F. Leymann. Service-oriented computing: a research roadmap. Int. J. Cooperative Inf. Syst., 17(2):223-255, 2008.

[13] T. J. Ross, J. M. Booker, and W. J. Parkinson. Fuzzy logic and probability applications: bridging the gap. American Statistical Association and Society for Industrial and Applied Mathematics, 2002.

[14] U. Wahli, O. Burroughs, O. Cline, A. Go, and L. Tung. Web Service Handbook for Websphere Application Server Version 6.1. IBM Corporation, 2006.

[15] Y. Wang and L. Li. Trust rating aggregations in serviceoriented applications. IEEE Trans. Services Computing, In press, 2011.

[16] Y. Wang and M. P. Singh. Formal trust model for multiagent systems. In International Joint Conference on Artificial Intelligence (IJCAI 2007), pages 1551-1556, 2007.

[17] J. Xiao and R. Boutaba. QoS-aware service composition and adaptation in autonomic communication. IEEE Journal on Selected Areas in Communications, 23(12):2344-2360, 2005.

[18] L. Xiong and L. Liu. PeerTrust: Supporting reputationbased trust for peer-to-peer electronic communities. IEEE Trans. Knowl. Data Eng., 16(7):843-857, 2004.

[19] Z. Xu, P. Martin, W. Powley, and F. Zulkernine. Reputationenhanced QoS-based web services discovery. In ICWS 2007, pages 249-256, 2007.

[20] B. Yu, M. P. Singh, and K. Sycara. Developing trust in large-scale peer-to-peer systems. IEEE Symposium on Multi-Agent Security and Survivability, pages 1-10, 2004.

[21] L. Zeng, B. Benatallah, A. H. H. Ngu, M. Dumas, J. Kalagnanam, and H. Chang. QoS-aware middleware for web services composition. IEEE Trans. Software Eng., 30(5):311-327, 2004. 